Honam Mathematical J. 33 (2011), No. 4, pp. 495-498

http://dx.doi.org/10.5831/HMJ.2011.33.4.495

\title{
A CONVERSE OF EULER'S THEOREM FOR POLYHEDRA
}

\author{
Seung-Ho Ahn, Dong-Soo Kim And Young Ho Kim
}

\begin{abstract}
We give a converse of the well-known Euler's theorem for convex polyhedra.
\end{abstract}

Let $\mathbf{D}$ be the set of all convex polyhedra and $\Phi: \mathbf{D} \rightarrow N^{3}$ the map defined by $\Phi(S)=(v, e, f)$, where $N$ denotes the set of all natural numbers, $v, e$, and $f$ the number of vertices, edges, and faces of a convex polyhedron $S$, respectively. Then the well-known Euler's theorem for polyhedra states that the image $\operatorname{Im} \Phi$ of $\Phi$ is contained in the plane

$$
\Pi=\left\{(v, e, f) \in N^{3} \mid v-e+f=2\right\} .
$$

A number of proofs of this theorem are presented in [2]. A heuristic proof may be also found in [4]. For a brief history of the theorem, see [1].

Obviously, $\operatorname{Im} \Phi$ is a proper subset of the plane $\Pi$. Hence it is natural to ask the following:

"For what values of $(v, e, f) \in \Pi$ does there exist a convex polyhedron $S$ with $\Phi(S)=(v, e, f) ? "$

In this short note, we give a complete answer to this question. More precisely, we shall give the following:

Theorem. The image of the map $\Phi: \mathbf{D} \rightarrow N^{3}$ is given by

$$
\operatorname{Im} \Phi=\left\{(v, e, f) \in N^{3} \mid v-e+f=2,2 e \geq 3 f, f \geq 4\right\} .
$$

First we give

Lemma 1. Let $v, e$, and $f$ denote the number of vertices, edges, and faces of a convex polyhedron $S$, respectively. Then we have

$$
2 e \geq 3 f,
$$

Received August 31, 2011. Accepted September 26, 2011.

2000 Mathematics Subject Classification. 52B05, 53B45.

Key words and phrases. Euler's theorem, convex polyhedra. 
where the equality holds in (1) if and only if each face is a triangle.

Proof. We give a proof for completeness. Let $f_{n}$ stand for the number of those faces that have precisely $n$ sides, then we have

$$
f=\sum_{n=3}^{\infty} f_{n}
$$

Furthermore, since every edge of the polyhedron is a side of exactly two faces, we also obtain

$$
2 e=\sum_{n=3}^{\infty} n f_{n} .
$$

Then, (2) and (3) imply the following inequality:

$$
2 e=\sum_{n=3}^{\infty} n f_{n} \geq 3 \sum_{n=3}^{\infty} f_{n}=3 f,
$$

which completes the proof of (1). The equality condition of (1) follows immediately from (4).

Now we define three maps $g_{i}: N^{3} \rightarrow N^{3}, i=1,2,3$ as follows:

$$
\begin{aligned}
& g_{1}(v, e, f)=(v+1, e+1, f), \\
& g_{2}(v, e, f)=(v+1, e+2, f+1), \\
& g_{3}(v, e, f)=(v+1, e+3, f+2) .
\end{aligned}
$$

Then we have

Lemma 2. Each map $g_{i}, i=1,2,3$ maps $\operatorname{Im} \Phi$ into $\operatorname{Im} \Phi$.

Proof. For any $(v, e, f) \in \operatorname{Im} \Phi$, we consider a convex polyhedron $S$ in $\mathbf{D}$ with $\Phi(S)=(v, e, f)$. Fix a convex $n$-gonal face $\sigma=P_{1} P_{2} \cdots P_{n}$ of the polyhedron $S$ where $P_{1}, P_{2}, \cdots, P_{n}$ denote the consecutive vertices of the face $\sigma$. Then, without loss of generality, we may assume that the straight line $\overleftrightarrow{P_{1} P_{2}}$ does not pass through $P_{3}$. Choose an interior point $P$ of the edge $P_{1} P_{2}$ and an interior point $Q$ of the triangle $P_{1} P_{2} P_{3}$.

We now construct a polyhedron $S_{1}$ consisting of the vertices as $P$ and all of vertices of $S$, the edges as $P_{1} P, P P_{2}$ and all edges of $S$ other than $P_{1} P_{2}$ and the faces as all of faces of $S$. Then $S_{1}$ is a convex polyhedron with $\Phi\left(S_{1}\right)=(v+1, e+1, f)$. Next, we consider the polyhedron $S_{2}$ consisting of the vertices as $P$ and other vertices of $S$, the edges as $P_{1} P, P P_{2}, P P_{3}$ and all edges of $S$ other than $P_{1} P_{2}$ and the faces as $P_{1} P P_{3} \cdots P_{n}, P P_{2} P_{3}$ and all of faces of $S$ except $\sigma$. Then $S_{2}$ is a convex 
polyhedron with $\Phi\left(S_{2}\right)=(v+1, e+2, f+1)$. Finally, we construct the polyhedron $S_{3}$ which consists of the vertices as $Q$ and the vertices of $S$, the edges as $P_{1} Q, P_{2} Q, P_{3} Q$ and all of edges of $S$ and the faces as $P_{1} Q P_{3} \cdots P_{n}, P_{1} P_{2} Q, P_{2} P_{3} Q$ and all of faces of $S$ other than $\sigma$. We see that $S_{3}$ is a convex polyhedron with $\Phi\left(S_{3}\right)=(v+1, e+3, f+2)$.

From (5) we see that each map $g_{i}, i=1,2,3$ satisfies $g_{i}(v, e, f)=$ $\Phi\left(S_{i}\right)$, which completes the proof.

We now prove our theorem. Suppose that $(v, e, f) \in N^{3}$ satisfies the following:

$$
v-e+f=2,
$$

and

$$
2 e \geq 3 f, \quad f \geq 4 .
$$

First note that for a tetrahedron $\Sigma$ we have $\Phi(\Sigma)=(4,6,4)$.

Case 1 . If $v \geq f$, then $(6)$ shows that $(v, e, f)$ satisfies the following:

$$
(v, e, f)-(4,6,4)=(v-f)(1,1,0)+(f-4)(1,2,1) .
$$

Let $m$ and $n$ denote the integers $v-f$ and $f-4$, respectively. Then (7) implies that $m$ and $n$ are nonnegative integers. (8) shows that

$$
(v, e, f)=g_{2}^{n} \circ g_{1}^{m}(4,6,4) .
$$

Case 2. If $v<f$, then $(6)$ shows that $(v, e, f)$ satisfies the following:

$$
(v, e, f)-(4,6,4)=(2 e-3 f)(1,2,1)+(f-v)(1,3,2) .
$$

Let $m$ and $n$ denote the integers $2 e-3 f$ and $f-v$, respectively. Then (7) implies that $m, n$ are nonnegative integers. (10) implies that

$$
(v, e, f)=g_{3}^{n} \circ g_{2}^{m}(4,6,4) .
$$

Thus together with (9) and (11), Lemma 2 shows that $\Phi(S)=(v, e, f)$ for a convex polyhedron $S$, which can be constructed from a tetrahedron $\Sigma$. This together with Lemma 1 completes the proof of our theorem.

Remark. In [3], they prove as follows that Euler characteristic $\chi=$ $v-e+f$ for polyhedra is the essentially unique topological invariant: Consider a map $g: \mathbf{D} \rightarrow R$ given by $g(S)=g(v, e, f)$, where $\Phi(S)=$ $(v, e, f)$. Suppose that $g$ is topologically invariant. Then $g$ is a function of $\chi=v-e+f$. 


\section{References}

[1] Hilton, P. and Pedersen, J., The Euler characteristic and Polya's dream, Amer. Math. Monthly 103(1996), 121-131.

[2] Hopf, H., Differential Geometry in the Large, Springer-Verlag, Berlin, 1983.

[3] Pak, C. R., Kim, S.-B., Kim, D.-S. and Cho, K. I., Are there any numbers other than the Euler number for polyhedra? (Korean), Comm. Korean Math. Soc. 15 (2000), no. 4, 669-674.

[4] Polya, G., Mathematical Discovery, Combined Edition, John Wiley and Sons, Inc., New York, 1981.

Seung-Ho Ahn

Department of Mathematics, Chonnam National University, Kwangju 500-757, Korea.

E-mail: shahn@chonnam.ac.kr

\section{Dong-Soo Kim}

Department of Mathematics, Chonnam National University, Kwangju 500-757, Korea.

E-mail: dosokim@chonnam.ac.kr

\section{Young Ho Kim}

Department of Mathematics, Kyungpook National University, Taegu 702-701, Korea.

E-mail: yhkim@knu.ac.kr 